\title{
An Improved Blind Color Image Watermarking using DCT in RGB Color Space
}

\author{
Jaya Jeswani \\ Lecturer \\ IT Department \\ Xavier Institute of Engineering \\ Mahim, Mumbai, India
}

\author{
Tanuja Sarode, Ph. D \\ Associate Professor \\ Computer Department \\ ThadomalShahani EngineeringCollege, Bandra, \\ Mumbai, India
}

\begin{abstract}
In this paper a novel blind watermarking technique for color images using DCT has been proposed. Inproposed technique cover image is decomposed into 3 planes, namely R, G, B and for watermarking B plane is selected. B plane is divided into blocks of size $8 \times 8$. DCT is applied to each $8 \times 8$ sized block and middle frequency coefficients are selected for embedding watermark. Finally binary watermark is embedded into each $8 \times 8$ DCT block by adjusting middle frequency coefficients DCT $(4,3)$ and DCT $(5,2)$. Experimental results shows that the proposed technique is imperceptible as well as robust against wide variety of attacks like Gaussian noise, Salt -pepper noise, Gaussian filter, Median filter, Histogram equalization etc and achieves average PSNR as $58.90 \mathrm{~dB}$. Proposed technique does not require cover image at the time of watermark extraction then too watermark is extracted completely and better than its counterparts available.
\end{abstract}

\section{General Terms}

Image Processing, Digital Watermarking,Watermark Embedding, Watermark Extracting

\section{Keywords}

DCT(Discrete Cosine Transform), MSE(Mean Square Error),NC (Normalized correlation), PSNR(Peak Signal to Noise)

\section{INTRODUCTION}

There is a need of some technique which allows protecting a digital data from illegal copying on web. Security can be provided with the help of digital watermarking [1]. Digital Watermarking is a technology in which there is embedding of copyright information in digital content which has to be protectedfrom illegal copying. So, in the proposed technique an application is developed for securely applying a binary watermark in color images, which provides ownership authentication to the end users.Digital watermarking is classified into two main categories namelyspatial domain andfrequency domain watermarking.Spatial domain watermarking refers to directly modify pixel values of an image to embed watermark. In frequency domain watermarking technique image is first transformed using appropriate transformation technique and then watermark is embedded into transformed coefficients of image. Frequency domain watermarking is complex and robust as compared to spatial domain techniques.Security and capacity of watermark data are very important issues to be considered.

A good watermarking technique should provide following fivefunctionalities:
Imperceptibility: The embedded watermarks are imperceptible both perceptually as well as statistically. The watermarks do not create visible artifacts in still images $[1,2$, and 3].

Inseparability: After the digital content is embedded with watermark, separating the content from the watermark to retrieve the original content is not possible $[4,5,6,7]$.

Robustness: The watermarks should not get degraded or destroyed as a result of unintentional or malicious signal and geometric distortions [8, 9, and 10].

Security: The digital watermarking techniques prevent unauthorized users from detecting and modifying the watermark embedded in the cover signal $[8,11,12,13]$.

Effectiveness: It means that the watermark extraction process should be simple and fast $[14,15,16,17,18,19,20]$.

In literature DCT transform has been successfully used for watermarking. DCT domain watermarking is classified into two types: Global DCT watermarking and Block-based DCT watermarking. In the Global DCT watermarking, the DCT computation is performed on the whole image, while in the Block-based DCT the image is divided into non-overlapping blocks and DCT computation is performed on each block separately to obtain low-frequency, mid-frequency and highfrequency sub-bands. Many watermarking techniques have been proposed using block based DCT and embeds watermark in different regions namely, Low Frequency, Mid-Band Frequency and High Frequency.S. Feng, D. Lin, S. C. Shie, J. Y. Guo, proposed a DCT-based technique, they converted RGB space to YUV space and embedded watermark in Y component [10].MaliheSoleimani, FaezehSanaeiNezhad, HadiMahdipour proposed DCT watermarkingtechnique using spread spectrum.They modified low frequency coefficient $(2,1)$ and $(1,2)$ and the bias member for embedding of binary watermark[16].Sudhanshu Gonge and JagdishBakal have proposed watermark embedding in middle frequency coefficients of DCT [17]. M. Yesilyurt, Y. Yalman,A. T. Ozcerit have proposed block based DCT and modified middle frequency components luminance component [18].

The organization of the paper is as follows. Section 2 describes theory of DCT, Section 3 describes coefficient selection, watermark embedding and watermark extracting algorithm, Section 4 discusses experimental results before and after applying image processing attacks, Section 5 concludes the paper. 


\section{DISCRETE COSINE TRANSFORM}

Discrete-Cosine-Transform (DCT) $[7,14,15,18,19]$ is a popular transform domain watermarking technique. DCT divides image into three frequency bands low frequency, middle frequency and high frequency sub -bands. Fig. 1shows DCT bands, $F_{L}$ denotes low frequency sub-band, $F_{M}$ denotes middle frequency sub-band, $\mathrm{F}_{\mathrm{H}}$ denotes the high frequency sub-band

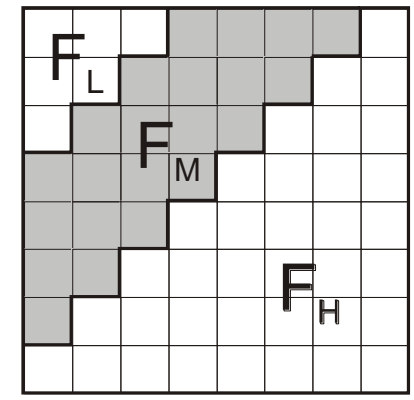

Fig. 1: DCT Bands [19]

The definition of 2-D DCT can be given as follows:

$C(u, v)=\alpha(u) \alpha(v) \sum_{x=0}^{N-1} \sum_{y=0}^{N-1} f(x, y) \cos \left[\frac{(2 x+1) u \pi}{2 N}\right] \cos \left[\frac{(2 y+1) v \pi}{2 N}\right](1)$

For $u, v=0, I, 2, \ldots, N-1$

The definition of 2-D inverse DCT can be given as follows,

$f(\mathrm{x}, \mathrm{y})=\sum_{u=0}^{N-1} \sum_{v=0}^{N-1} \alpha(\mathrm{u}) \boldsymbol{\alpha}(\mathrm{v}) \mathrm{C}(\mathrm{u}, \mathrm{v}) \cos \left[\frac{(2 x+1) u \pi}{2 N}\right] \cos \left[\frac{(2 y+1) v \pi}{2 N}\right](2)$ For

$x, y=0,1,2, \ldots, N-1$

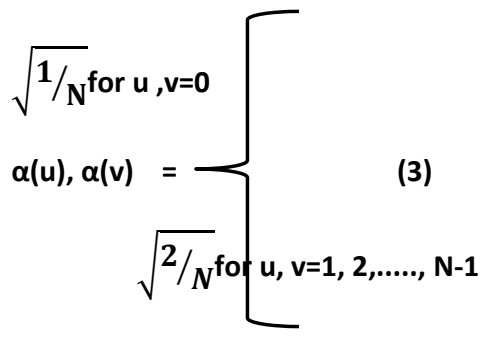

In this paper for watermark embedding middle frequency bands are chosen because low frequency band contains more of the energy content of carrier image, embedding the watermark in low frequency affects the quality of watermarked image and embedding watermark in high frequency gets removed through noise attacks.

\section{PROPOSED METHOD}

Transform domains are better for the watermarking than spatial domain for both reasons of robustness as well as visual quality of watermarked image $[1,2,3,5,18,19,20]$.

\subsection{Coefficient Selection}

For watermark embedding DCT $(4,3)$ and $\operatorname{DCT}(5,2)$ have been selected because both are middle frequency components and in JPEG quantization tableboth are having same value as 22. The choice in selecting the two locations is dependent on the content of the JPEG quantization table given in table 1. The two locations which have identical quantization values are selected for embedding one watermark bit of information.From the table the coefficients at $(5,2)$ and $(4,3)$ have value 22 or $(2,3)$ and $(4,1)$ have value 14 , would make suitable candidates for comparison, as their quantization values are equal. Fig. 2 below shows division of image into block size $8 \times 8$.

\begin{tabular}{|l|l|l|l|l|l|l|l|}
\hline$(1,1)$ & $(1,2)$ & $(1,3)$ & $(1,4)$ & $(1,5)$ & $(1,6)$ & $(1,7)$ & $(1,8)$ \\
\hline$(2,1)$ & $(2,2)$ & $(2,3)$ & $(2,4)$ & $(2,5)$ & $(2,6)$ & $(2,7)$ & $(2,8)$ \\
\hline$(3,1)$ & $(3,2)$ & $(3,3)$ & $(3,4)$ & $(3,5)$ & $(3,6)$ & $(3,7)$ & $(3,8)$ \\
\hline$(4,1)$ & $(4,2)$ & $(4,3)$ & $(4,4)$ & $(4,5)$ & $(4,6)$ & $(4,7)$ & $(4,8)$ \\
\hline$(5,1)$ & $(5,2)$ & $(5,3)$ & $(5,4)$ & $(5,5)$ & $(5,6)$ & $(5,7)$ & $(5,8)$ \\
\hline$(6,1)$ & $(6,2)$ & $(6,3)$ & $(6,4)$ & $(6,5)$ & $(6,6)$ & $(6,7)$ & $(6,8)$ \\
\hline$(7,1)$ & $(7,2)$ & $(7,3)$ & $(7,4)$ & $(7,5)$ & $(7.6)$ & $(7,7)$ & $(7,8)$ \\
\hline$(8,1)$ & $(8,2)$ & $(8,3)$ & $(8,4)$ & $(8,5)$ & $(8,6)$ & $(8,7)$ & $(8,8)$ \\
\hline
\end{tabular}

Fig. 2:Coefficient Selection in a $8 \times 8$ DCT block

Table1.JPEG Quantization Table

\begin{tabular}{|l|l|l|l|l|l|l|l|}
\hline 16 & 11 & 10 & 26 & 24 & 40 & 51 & 61 \\
\hline 12 & 12 & 14 & 19 & 26 & 58 & 60 & 55 \\
\hline 14 & 13 & 16 & 24 & 40 & 57 & 69 & 56 \\
\hline 14 & 17 & 22 & 29 & 51 & 87 & 80 & 62 \\
\hline 18 & 22 & 37 & 56 & 68 & 109 & 103 & 77 \\
\hline 24 & 35 & 55 & 64 & 81 & 104 & 113 & 92 \\
\hline 49 & 64 & 78 & 87 & 103 & 121 & 120 & 101 \\
\hline 72 & 92 & 95 & 98 & 112 & 100 & 103 & 99 \\
\hline
\end{tabular}

\subsection{Watermark Embedding Algorithm}

Inputs: Color cover image and binary watermark image

Outputs: Watermarked image

The steps are as follows:

1. Take cover image of size $\mathrm{M} \times \mathrm{N}$ and binary watermark of sizen $\times n$ as input.

2. Decompose the image into three color planes: $\mathrm{R}, \mathrm{G}$ and $\mathrm{B}$.

3. Select $B$ plane and divide it into $8 \times 8$ sized blocks.

4. Determine maximum watermark size based on cover image and block size by :

max_watermark_size $=\mathrm{N} \times \mathrm{N} /$ block $\operatorname{size}^{2}(4)$

5. Check that the watermark isn't too large for cover image.Pad the watermark out to the maximum watermark size with ones.

6. Apply DCT on every $8 \times 8$ block of B plane.

7. Encodeswatermark bit $=0$ whenDCT $(5,2) \quad>=$ $\operatorname{DCT}(4,3)$

8. Encodeswatermark bit=1 when $\operatorname{DCT}(5,2)<$ DCT $(4,3)$.

9. If watermark bit $=0$ and $\operatorname{DCT}(5,2)<\operatorname{DCT}(4,3)$ then we need to swap them.

10. If watermark bit $=1$ and $\operatorname{DCT}(5,2)>=\operatorname{DCT}(4,3)$ and then we need to swap them.

11. Adjust the two values such that their difference $=\mathrm{k}$, where $\mathrm{k}$ is a secret key for watermark embedding process, which is known asminimum coefficient difference of DCT blocks. 
12. Transform block back into spatial domain by IDCT which gives watermarked image.

13. Combine R,G and modified $B$ plane to get watermarked Image.

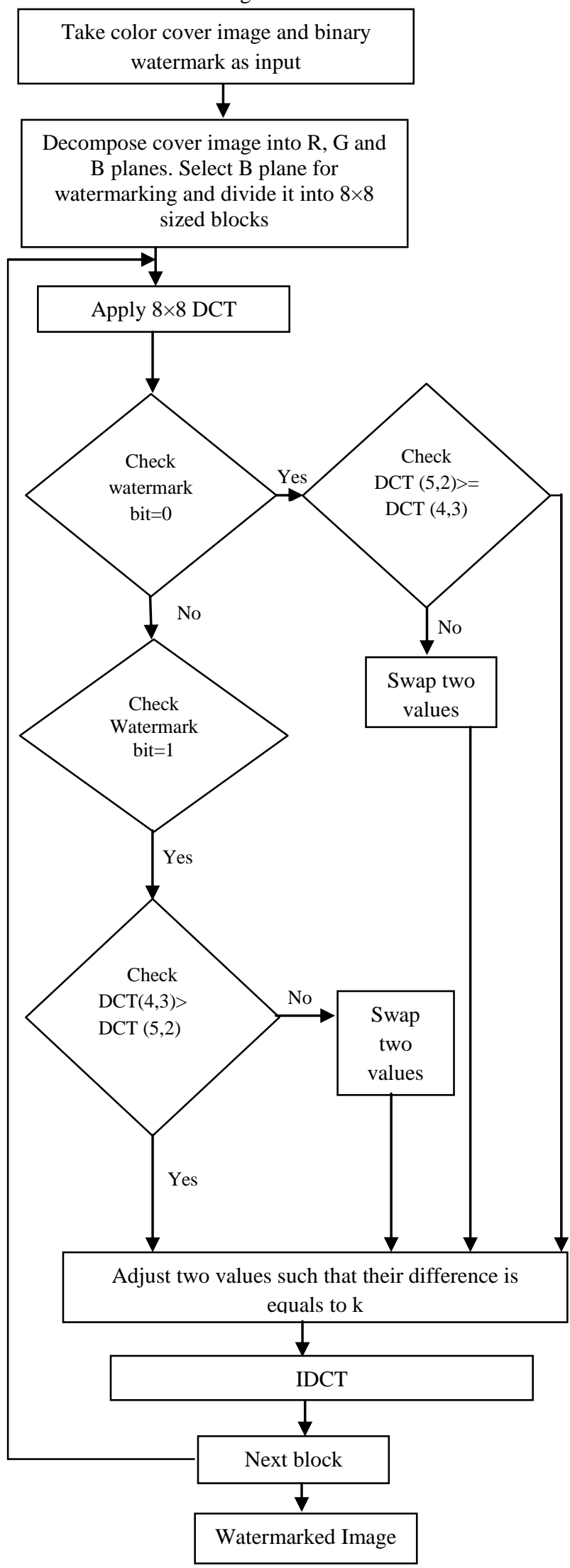

Fig. 3: Flow chart of Watermark Embedding Algorithm

\subsection{Watermark Extracting Algorithm}

Input: Watermarked image

Output:Extracted binary watermark

The steps are as follows:

1. Take watermarked image of size $\mathrm{M} \times \mathrm{N}$ as an input.

2. Decompose watermarked image into 3 planes: R,G and $\mathrm{B}$.

3. Select $\mathrm{B}$ plane and divide it into $8 \times 8$ sized blocks

4. Apply DCT on every $8 \times 8$ block of B plane.

5. If $\operatorname{DCT}(5,2)>=\operatorname{DCT}(4,3)$, make watermark bit $=0$ else watermark bit $=1$.

6. Reshape the recovered binary watermark into $n \times n$.

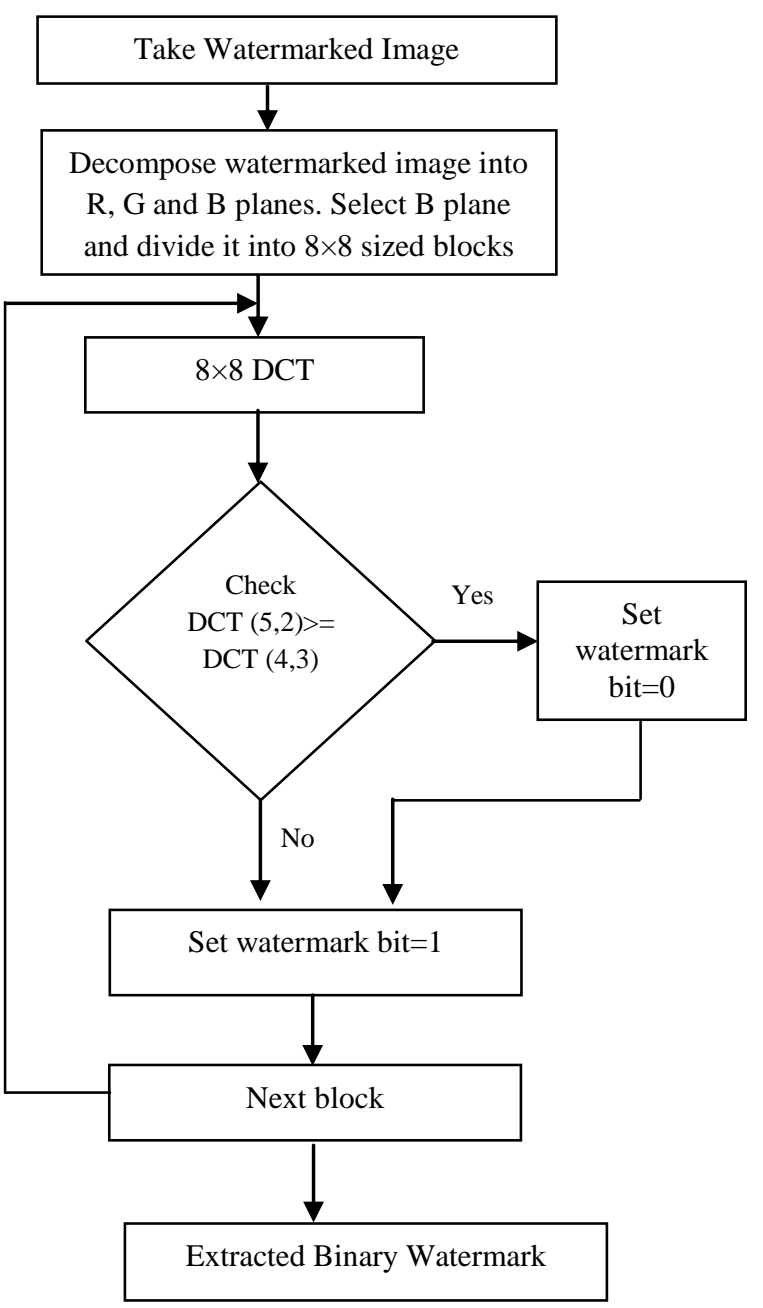

Fig. 4: Flow chart of Watermark Extraction Algorithm

Fig.3 and Fig.4 shows flow charts of proposed method watermark embedding and extraction process respectively.

\section{EXPERIMENTAL RESULTS}

For experimentalpurpose nine $512 \times 512$ colour cover images namely Waterlili, Roses,House, Madhuri, Macaw, Mom, Candle, Fish, Horse and $64 \times 64$ (8.bmp) binary watermark are used. For evaluating the performance of proposed techniquePSNR, MSE and NC are used and shown in 
equation 4, 5 and 6 respectively.Fig. 5 shows nine test cover images. Fig.6 and Fig.7 shows watermark embedding and

The Peak Signal to Noise Ratio (PSNR) is used to measure deviation of the watermarked image and attacked image from the original image,

$\mathrm{MSE}=\frac{1}{\mathrm{MN}} \sum_{\mathbf{i}=1}^{\mathrm{M}} \sum_{\mathbf{j}=1}^{\mathrm{N}}\left[\mathrm{I}(\mathbf{i}, \mathbf{j})-\mathrm{I}^{\prime}(\mathbf{i}, \mathbf{j})\right]^{2}(\mathbf{4})$

Where,

$\mathrm{M}, \mathrm{N}=$ size of the original image,

$I(i, j)=$ pixel values at location $(i, j)$ of the original image,

$I^{\prime}(i, j)=$ pixel values at location $(i, j)$ of watermarked image

To obtain PSNR value following equation will be used,

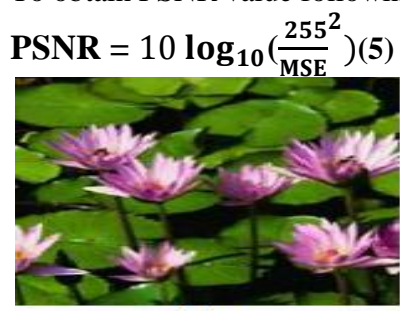

(a)

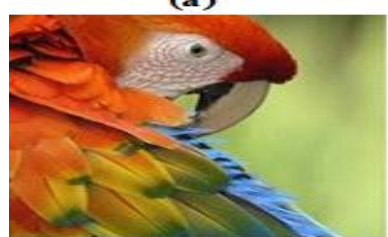

(e)

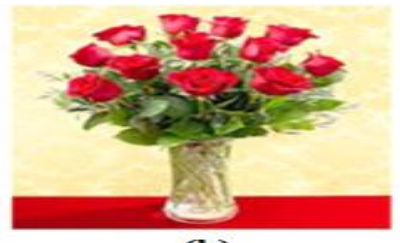

(b)

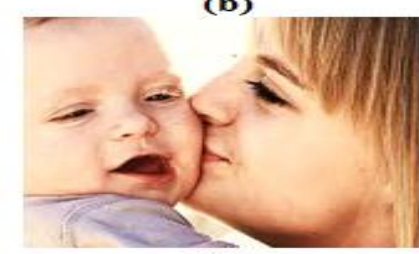

(f)

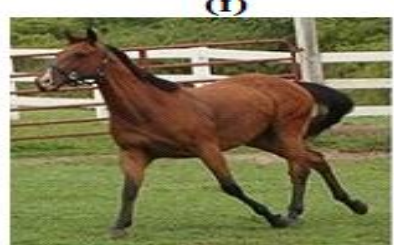

(i) watermark extraction

onwaterlili image respectively.

The normalized correlation (NC) gives a measure of the robustness of watermarking and its peak value is one.

$\mathbf{N C}=\frac{\sum_{\mathrm{i}} \sum_{\mathrm{j}} \mathbf{w}(\mathbf{i}, \mathbf{j}) \mathbf{w}^{\prime}(\mathbf{i}, \mathbf{j})}{\sum_{\mathbf{i}} \sum_{\mathbf{j}} \mathbf{w}(\mathbf{i}, \mathbf{j})^{2}}(\mathbf{6})$

$\mathrm{W}(\mathrm{i}, \mathrm{j})=$ pixel values at location $(\mathrm{i}, \mathrm{j})$ of the original watermark,

$W^{\prime}(i, j)=$ pixel values at location $(i, j)$ of the extracted watermark

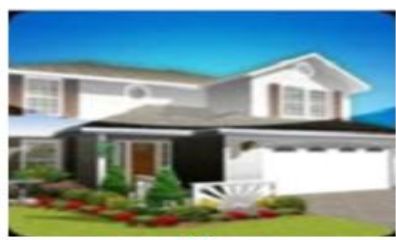

(c)
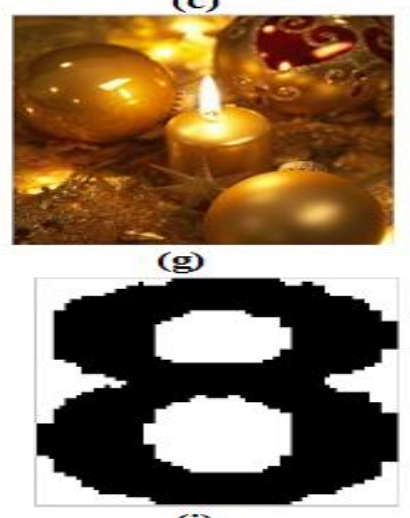

(i) (d)

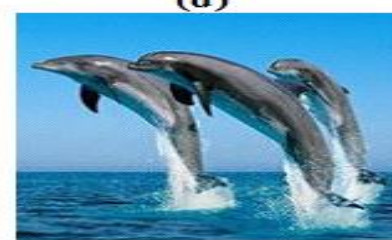

(h)

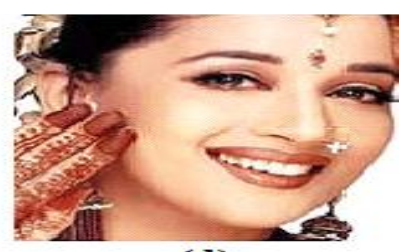

Fig. 5:Test Cover Images (a) Waterlili(b) Roses (c)House (d) Madhuri (e) Macaw (f) Mom (g) Candle (h) Fish (i) Horse (j) 8 (Binary watermark)

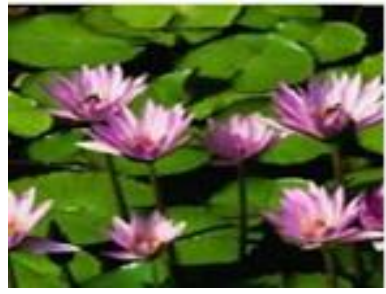

Original Image

$(512 \times 512)$

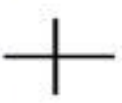

Binary Watermark

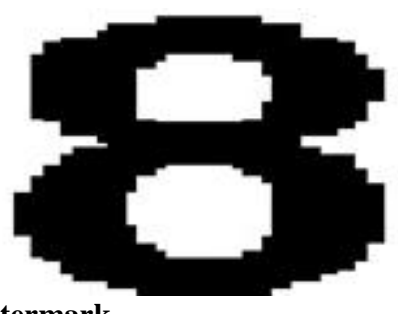

$(64 \times 64)$

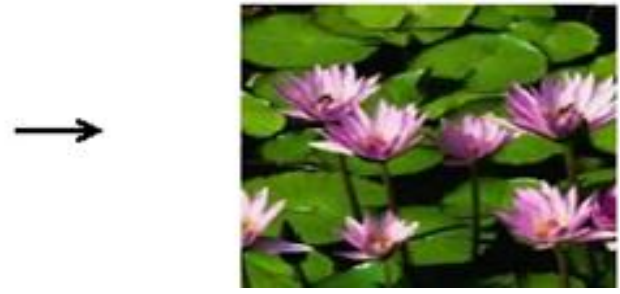

Watermarked Image

$(512 \times 512)$

Fig. 6 Watermark Embedding Process
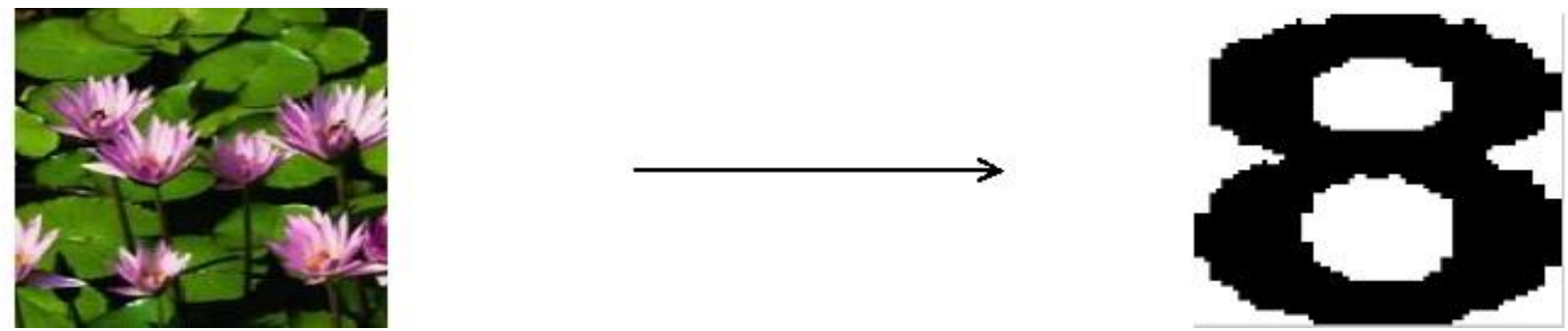

Watermarked ImageRecovered Watermark $(512 \times 512)$

(64×64)

Fig. 7: Watermark Extraction Process 
Table 2. PSNR and NC values of watermark embedding and extraction onwaterlili image at different values of $k$

\begin{tabular}{|c|c|c|c|}
\hline $\begin{array}{c}\text { DC } \\
\text { Coefficient } \\
\text { Difference k }\end{array}$ & PSNR & MSE & NC \\
\hline 1 & 61.9932 & 0.0414 & 0.4853 \\
\hline 2 & 61.4307 & 0.0471 & 0.8784 \\
\hline $\mathbf{3}$ & $\mathbf{6 0 . 2 8 5 6}$ & $\mathbf{0 . 0 6 0 9}$ & $\mathbf{1}$ \\
\hline 4 & 59.3649 & 0.0758 & 1 \\
\hline 5 & 58.1898 & 0.0994 & 1 \\
\hline 6 & 57.3715 & 0.1200 & 1 \\
\hline 7 & 56.5813 & 0.1439 & 1 \\
\hline 8 & 55.8277 & 0.1712 & 1 \\
\hline 9 & 55.2251 & 0.1967 & 1 \\
\hline 10 & 54.6747 & 0.2233 & 1 \\
\hline
\end{tabular}

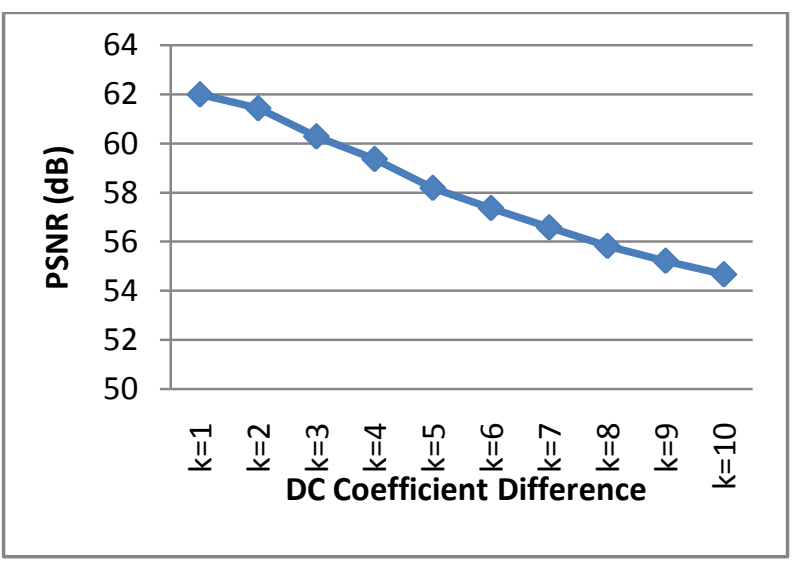

(a)

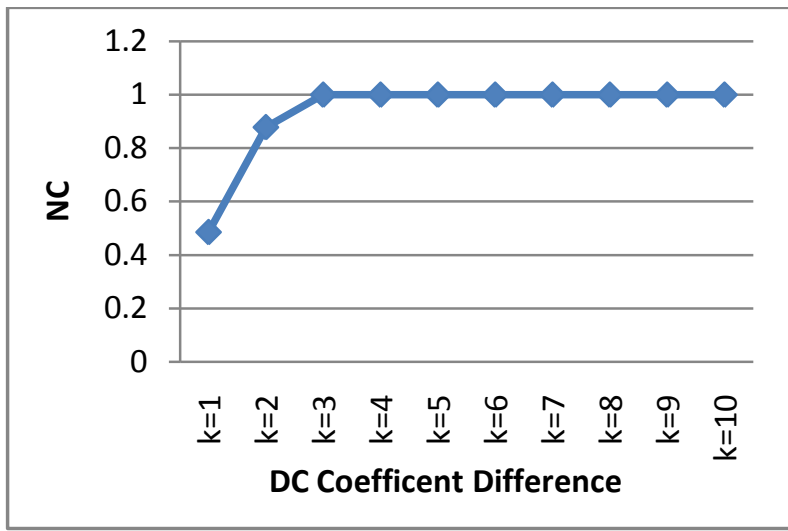

(b)

Fig.8: (a) Peak Signal to Noise Ratio Chartof waterlili image at different values of $k(b)$ Mean Square Error chartof waterlili image at different values of $k$
In the proposed algorithm $\mathrm{k}$ is DC coefficient difference between DCT $(5,2)$ and DCT $(4,3)$ and known as a secrete key for watermark embedding. Different values of $\mathrm{k}$ are tried on waterlili image. From Table 2 it can be seen that as the value of $\mathrm{k}$ increases, value of PSNR decreases and $\mathrm{NC}$ value increases till certain value of $\mathrm{k}$ and after reaching that value of $\mathrm{k}, \mathrm{NC}$ value doesn't change.Fig. 8 shows PSNR and NC chart of waterlili image at different values of $k$. Value of $k=3$ is selected for waterlili image because it gives optimal results and value of $\mathrm{k}$ varies from image to image. When PSNR is higher than 30 , it is considered that watermarked image has a very good quality. Moreover when NC value is higher than 0.75 , the extracted watermark is considered as valid one. Table 3 shows that the average PSNR achieved is 58.90 and NC value without applying any attack achieved is 1 .Table 4 shows experimental results of common image processing attacks like noise, filtering attacks etc. Table 5 shows results of different image processing attacks on all test cover images. Table 6 below shows comparison of proposed method with its counterparts available.

Table3.PSNR and NC values of watermark embedding process and extraction process on all test cover images

\begin{tabular}{|c|c|c|c|c|}
\hline $\begin{array}{c}\text { Sr. } \\
\text { No }\end{array}$ & Image & PSNR & MSE & NC \\
\hline 1 & Waterlili & 60.2856 & 0.0609 & 1 \\
\hline 2 & Roses & 59.1613 & 0.0789 & 1 \\
\hline 3 & House & 58.1161 & 0.1003 & 1 \\
\hline 4 & Madhuri & 59.6937 & 0.0698 & 1 \\
\hline 5 & Macaw & 59.1595 & 0.0789 & 1 \\
\hline 6 & Mom & 58.5090 & 0.0917 & 1 \\
\hline 7 & Candle & 57.9072 & 0.1053 & 1 \\
\hline 8 & Fish & 58.4812 & 0.0922 & 1 \\
\hline 9 & Horse & 58.8224 & 0.0853 & 1 \\
\hline
\end{tabular}

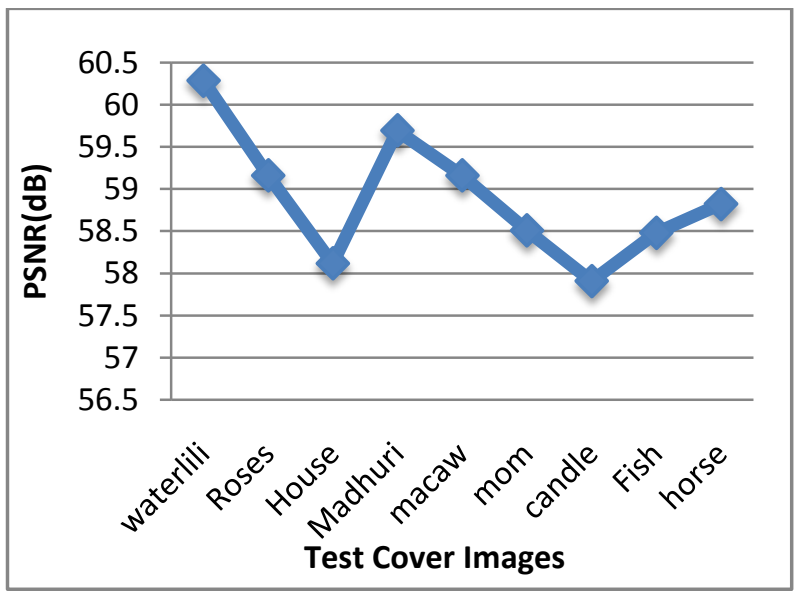

Fig.9: Peak Signal to Noise Ratio Chart of different test cover images 
Table 4.Experimental results of common image processing attacks on waterlili image

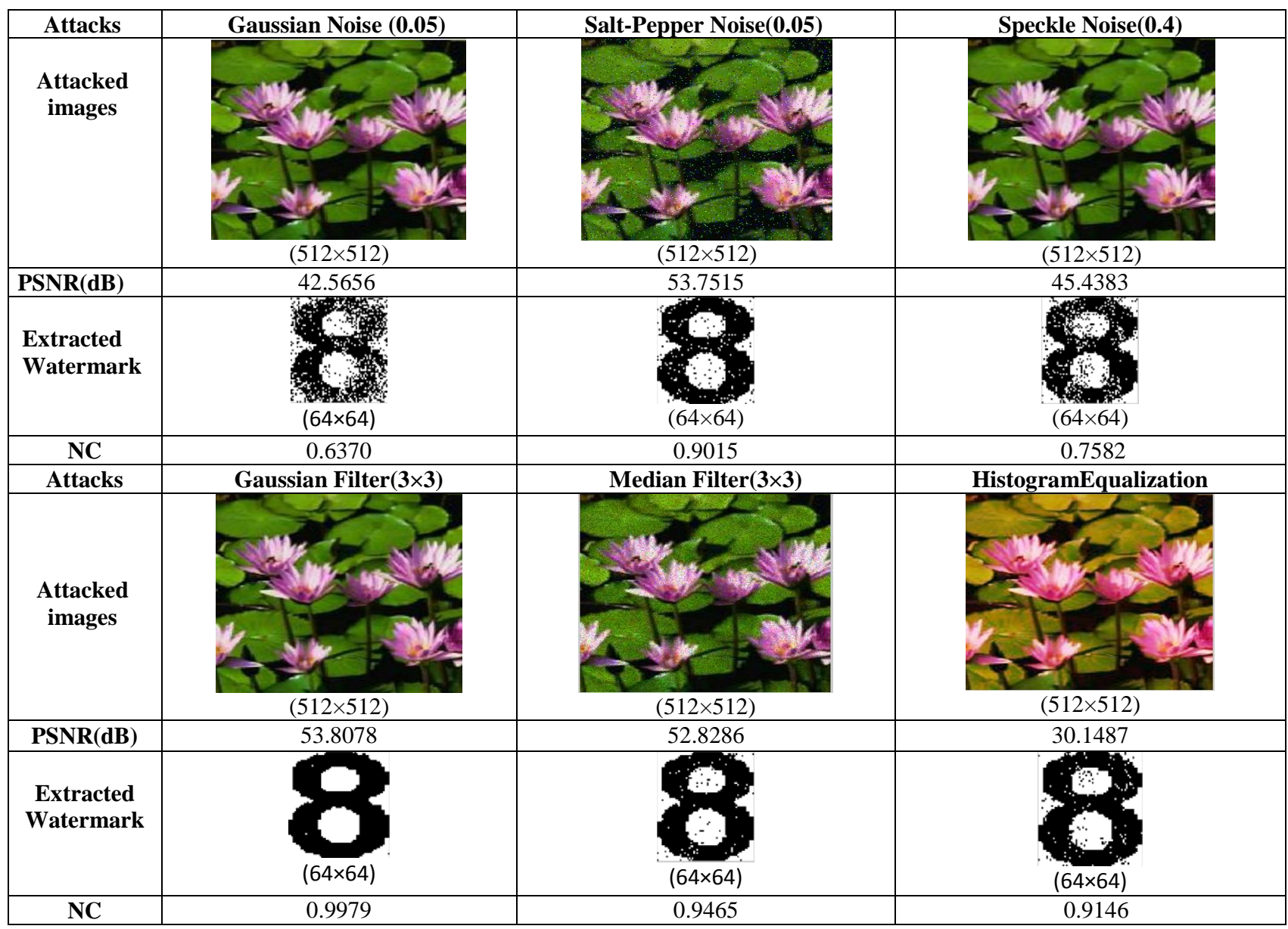

Table 5. Experimental results of common image processing attacks on all test cover images

\begin{tabular}{|c|c|c|c|c|c|c|c|c|c|c|}
\hline Attacks & $\begin{array}{c}\text { Performance } \\
\text { Evaluators }\end{array}$ & Waterlili & Roses & House & Madhuri & Macaw & Mom & Candle & Fish & Horse \\
\hline \multirow{2}{*}{$\begin{array}{c}\text { Gaussian Noise } \\
(\mathbf{0 . 0 5})\end{array}$} & PSNR (dB) & 42.5656 & 42.5280 & 42.8775 & 42.8996 & 42.8795 & 42.8213 & 42.9425 & 42.8538 & 42.8307 \\
\hline & $\mathbf{N C}$ & 0.6370 & 0.8476 & 0.8677 & 0.8623 & 0.8379 & 0.8553 & 0.9069 & 0.8607 & 0.8798 \\
\hline \multirow{2}{*}{$\begin{array}{c}\text { Salt-pepper } \\
\text { Noise } \\
(\mathbf{0 . 0 5}) \\
\end{array}$} & PSNR (dB) & 53.7515 & 49.5251 & 52.8013 & 52.6059 & 53.0267 & 52.7852 & 52.7611 & 52.4594 & 52.6239 \\
\hline & $\mathrm{NC}$ & 0.9015 & 0.8795 & 0.8841 & 0.9090 & 0.8456 & 0.8891 & 8860 & 0.8784 & 0.8726 \\
\hline \multirow{2}{*}{$\begin{array}{c}\text { Speckle Noise } \\
(0.4)\end{array}$} & PSNR (dB) & 45.4383 & 39.5407 & 41.3582 & 39.9736 & 41.8542 & 39.7528 & 44.0971 & 41.1102 & 43.1693 \\
\hline & $\mathrm{NC}$ & 0.7582 & 0.6434 & 0.8509 & 0.6655 & 0.7492 & 0.6385 & 0.8592 & 0.9027 & 0.8729 \\
\hline \multirow{2}{*}{$\begin{array}{c}\text { Gaussian } \\
\text { Filter } \\
(\mathbf{3} \times \mathbf{3})\end{array}$} & PSNR (dB) & 53.8078 & 43.7047 & 51.2728 & 49.4275 & 49.6612 & 49.5123 & 49.7182 & 50.0900 & 50.5612 \\
\hline & $\mathrm{NC}$ & 0.9979 & 0.9630 & 0.9593 & 0.9712 & 0.9322 & 0.9608 & 0.9927 & 0.9617 & 0.9748 \\
\hline \multirow{2}{*}{$\begin{array}{l}\text { Median } \\
\text { Filter } \\
(3 \times 3)\end{array}$} & PSNR (dB) & 52.8286 & 45.8905 & 50.1812 & 52.1383 & 55.8841 & 50.5974 & 50.0296 & 52.2714 & 53.6572 \\
\hline & $\mathrm{NC}$ & 0.9465 & 0.8454 & 0.8462 & 0.8846 & 0.8905 & 0.8981 & 0.9336 & 0.9184 & 0.9205 \\
\hline \multirow{2}{*}{$\begin{array}{l}\text { Histogram } \\
\text { Equalization }\end{array}$} & PSNR (dB) & 30.1487 & 30.2978 & 30.5771 & 30.2555 & 30.3830 & 31.0726 & 32.9705 & 33.8089 & 32.5771 \\
\hline & $\mathrm{NC}$ & 0.9146 & 0.8351 & 0.8692 & 0.8792 & 0.8848 & 0.9190 & 0.9635 & 0.9096 & 0.8692 \\
\hline
\end{tabular}


Table 6. PSNR results comparisons between proposed method and its counterparts

\begin{tabular}{|c|c|c|c|c|c|}
\hline Method & Domain & Color Model & PSNR(dB) & Cover Image & Watermark \\
\hline S. Feng et al. [10] & DCT & YUV (Y) & 33.90 & $512 \times 512$ & $32 \times 32$ \\
\hline Kaurand and Kaur [14] & DCT & YCbCr (Y) & 39.00 & $512 \times 512$ & $64 \times 64$ \\
\hline Yesilyurt, Yalman, Ozcerit [18] & DCT & YCbCr(Y) & 40.14 & $512 \times 512$ & $64 \times 64$ \\
\hline Proposed Method & DCT & RGB(B) & $\mathbf{5 8 . 9 0}$ & $\mathbf{5 1 2 \times 5 1 2}$ & $\mathbf{6 4 \times 6 4}$ \\
\hline
\end{tabular}

\section{CONCLUSION}

Many image watermarking algorithms have been proposed in spatial and frequency domains. Very few watermarking algorithm are proposed for color images. In this paper a nonblind robust color image watermarking is proposed in frequency domain using DCT.Proposed algorithm embeds binary watermark in color images. Experimental results shows that proposed method is imperceptible as well as robust.The robustness of the watermarking methods has been measured by computing the PSNR and NC values. Proposed method achieves average PSNR as $58.90 \mathrm{~dB}$ and better than its counterparts available. The proposed method is secure becausewatermark is embedded only on B plane. Thus, can be used as a non-blind color image watermarking algorithm.

\section{REFERENCES}

[1] Potdar, V. M., Han, S., and Chang, E., "A Survey of Digital Image Watermarking Techniques", 3rd IEEEInternational Conference on Industrial Informatics(INDIN), pp. 709-716, 2005.

[2] Chris Shoemakar, "Hidden Bits: A Survey of Techniques for Digital Watermarking” Independent StudyEER-290 Prof RudkoSpring 2002.

[3] Smitha Rao, Jyothsna A. N, Pinaka Pani. R, "Digital watermarking: applications, techniques and attacks", International Journal of Computer Applications Volume 44, No. 7, pp. 29-34, April 2012.

[4] Manik Mondal, Debalina Barik, "Spatial domain robust watermarking scheme for color image,"International Journal of Advanced Computer Science, vol.2, no.1, pp. 24-27,Jan 2012.

[5] N. Kaewkamnerd ,K.R. Rao, "Wavelet Based Image Adaptive Watermarking Scheme", IEEE Electronic Letters, Vol. 36, pp. 312-313, Feb.2000.

[6] Hui-fang, LI., Ning, C., and Xiao-ming, C., "A study on image digital watermarking based on wavelet transform", Journal of China Universities of Posts and Telecommunications, vol.17, pp. 122-126, July 2010.

[7] Rao K, P. Yip, "Discrete Cosine Transform: algorithms, advantages, applications", Academic press, USA, 1990.

[8] Masoumi, M., Amiri, S., "A High Capacity Digital Watermarking Scheme for Copyright protection of Video Data based on $\mathrm{YCbCr}$ Color Channels Invariant to Geometric and Non-Geometric Attacks", International Journal of Computer Applications, vol.51, no.13, pp. 0975-8887, August 2012.

[9] R. Eswaraiah \& E. Sreenivasa Reddy, "Robust Watermarking Method for Color Images Using DCT Coefficients of Watermark", Global Journals Inc.(US) 2012.

[10] S. Feng, D. Lin, S. C. Shie, J. Y. Guo, "Improving the robustness of DCT based image watermarking against
JPEG compression”, Computer Standards \& Interface, vol. 32, pp. 54-60, 2010.

[11] J. R. Hemandez, M. Amado, "DCT domain watermarking techniques for still images as detector performance analysis and a new structure," in IEEE Transactions on Image Processing, vol. 9, pp.5568,2000.

[12] Z. M. Zhang, L. Wang, "Semiblind image watermarking algorithm in DCT domain with chaotic encryption," in Computer Engineering, vol. 29, pp. 10,2003.

[13] Xijin, W., Linxiu, F., "The Application Research of MD5 Encryption Algorithm in DCT Digital Watermarking", International conference on Solid State Devices and Materials Science, Journal of Physics Procedia, vol.25, pp.1264-1269, 2012.

[14] M. Kaurand, P. Kaur, "Robust watermarking into the color models based on the synchronization template", in Proc. of the International Conference on Information and Multimedia Technology, pp.296-300, 2009.

[15] Dr. K. Ramanjaneyulu , Dr. P. Pandarinath and B. Rakesh Reddy, "Robust and Oblivious Watermarking based on Swapping of DCT Coefficients", International Journal of Application or Innovationin Engineering \& Management, vol. 2, issue 7, July 2013.

[16] Malihe Soleimani, Faezeh Sanaei Nezhad, Hadi Mahdipour, and Morteza Khademi "A Robust Digital Blind Image Watermarking Based on Spread Spectrum in DCT Domain",Science Academy Transactions on Computers and Communication Network (SATCCN) Vol. 2, No.2, June 2012, ISSN: 2046-5157

[17] Sudhanshu S.Gonge, Jagdish w. Bakal "Robust Digital Watermarking Technique by Using DCTAND Spread Spectrum",International Journal of Electrical, Electronics and Data Communication, ISSN (p): 23202084, Vol-1, Issue-2, April-2013.

[18] M. Yesilyurt, Y. Yalman, A. T. Ozcerit.,"A New DCT Based Watermarking Method Using Luminance Component",elektronika IR elektrtechnika, ISSN 13921215, Vol. 19, no. 4, 2013.

[19] Manish Choubisa, Kamal Hiran,S. K. Singh "Permutation Based Invisible Digital Watermarking Technique using DCT Domain", International Journal of Computer Applications (0975 - 8887) Vol. 31- no.6, October 2011.

[20] XU, Z. J., WANG, Z. Z., and LU, Q., "Research on Image Watermarking Algorithm based on DCT", 3rd International Conference on Environmental Science and Information Application Technology, Journal of Procedia Environmental Sciences, vol.10, pp. 11291135, 2011. 